\title{
ANATOMICAL COMPARISON OF THE RENAL ARTERIES IN THE RABBIT AND EUROPEAN HARE
}

\author{
Flešárová, S., Maženský, D. \\ Department of Anatomy, Histology and Physiology, \\ University of Veterinary Medicine and Pharmacy, Komenského 73, 04181 Košice \\ Slovakia
}

slavka.flesarova@uvlf.sk

\section{ABSTRACT}

The aim of this paper was to compare the level of origin of the renal arteries in the rabbit and hare. The study was carried out on ten adult rabbits and ten adult European hares using the corrosion cast technique. After the euthanasia, the vascular network was perfused with saline. Batson's corrosion casting kit No. 17 was used as a casting medium. After polymerization of the medium, the maceration was carried out in $\mathrm{KOH}$ solution. We found variable levels of the origin of renal arteries in the rabbit, in the hare and between both species. In the rabbit, the right renal artery originated at the level of the second lumbar vertebra in $70 \%$ of the cases and at the level of the first lumbar vertebra in $30 \%$ of the cases, and the left-sided renal artery originated in $60 \%$ of the cases at the level of the second lumbar vertebra and at the level of the third lumbar vertebra in $40 \%$ of the cases. In the hare, the bilateral renal arteries originated at the level of the second lumbar vertebra. According to the results, it can be concluded that the origin level of the renal arteries from the abdominal aorta is more variable in the domesticated rabbit in comparison with the hare.
Key words: abdominal aorta; corrosion cast; European hare; rabbit; renal artery

\section{INTRODUCTION}

Different species of mammals serve as experimental models for urologic procedures comprising the surgical and diagnostic techniques. The most frequently utilized species are the pig [7], dog [5] and rabbit [3, 18].

Knowledge of the variations of renal vascular anatomy play a significant role in exploration and treatment of renal transplantation, renal artery embolization, renal trauma, renovascular hypertension, angioplasty or vascular reconstruction for congenital and acquired lesions, conservative or radical renal surgery and surgery for abdominal aortic aneurysms.

The arterial arrangement of the kidneys has been the object of numerous anatomical studies in pigs $[2,12,13$, 19], dogs [4, 6, 14] and rabbits [17]. The urological literature still lacks significant knowledge concerning the renal arterial system of wild species. 
The aim of this study was to describe the origin, localization and variations of the renal arteries in two related species: the domesticated rabbit and European hare.

\section{MATERIALS AND METHODS}

This study was carried out on 10 adult European hares (Lepus Europaeus, L. 1758, age 140 days) and on 10 adult rabbits (Oryctolagus cuniculus $f$. domestica, L. 1758, age 140 days). We used hares (obtained from ISFA APRC, Nitra, Slovakia) of both sexes (female $n=5$; male $n=5$ ) with a weight range between $2.5-3.2 \mathrm{~kg}$, and New Zealand White rabbits (obtained from HYLAPA s. r. o., Prešov, Slovakia) of both sexes (female $n=5$; male $n=5$ ), in an accredited experimental laboratory of the University of Veterinary Medicine and Pharmacy in Košice, Slovakia. The animals were kept in cages under standard conditions (temperature $15-20^{\circ} \mathrm{C}$, relative humidity $45 \%$, 12-hour light period), and fed with a granular feed mixture (O-10 NORM TYP, Spišské kŕmne zmesi, Spišské Vlachy, Slovakia). The drinking water was available to all animals ad libitum. The animals were injected intravenously with heparin (50000 IU. $\mathrm{kg}^{-1}$ ) $30 \mathrm{~min}$ before they were sacrificed by intravenous injection of embutramide (T-61, $0.3 \mathrm{ml} . \mathrm{kg}^{-1}$ ). Immediately after euthanasia, the vascular network was perfused with a physiological solution. During manual injection through the ascending aorta, the right atrium of the heart was opened in order to lower the pressure in the vessels in order to ensure an optimal injection distribution. Batson's corrosion casting kit No. 17 using a volume of $50 \mathrm{ml}$ (Dione, České Budějovice, Czechia) was used as the casting medium. The maceration was carried out in $2-4 \% \mathrm{KOH}$ solution for a period of 5 days at $60-70{ }^{\circ} \mathrm{C}$. This study was carried under the authority decision No. 2647/07-221/5.

\section{RESULTS}

The renal arteries arising from the lateral surfaces of the abdominal aorta supply the kidneys. According to the more lateral position of the left kidney, the left renal artery was longer than the right one. In all of the cases, the right renal artery originated cranially to the left renal artery. Each renal artery was directed towards the renal hilus of the corresponding kidney. After it reached the renal hilus, it was

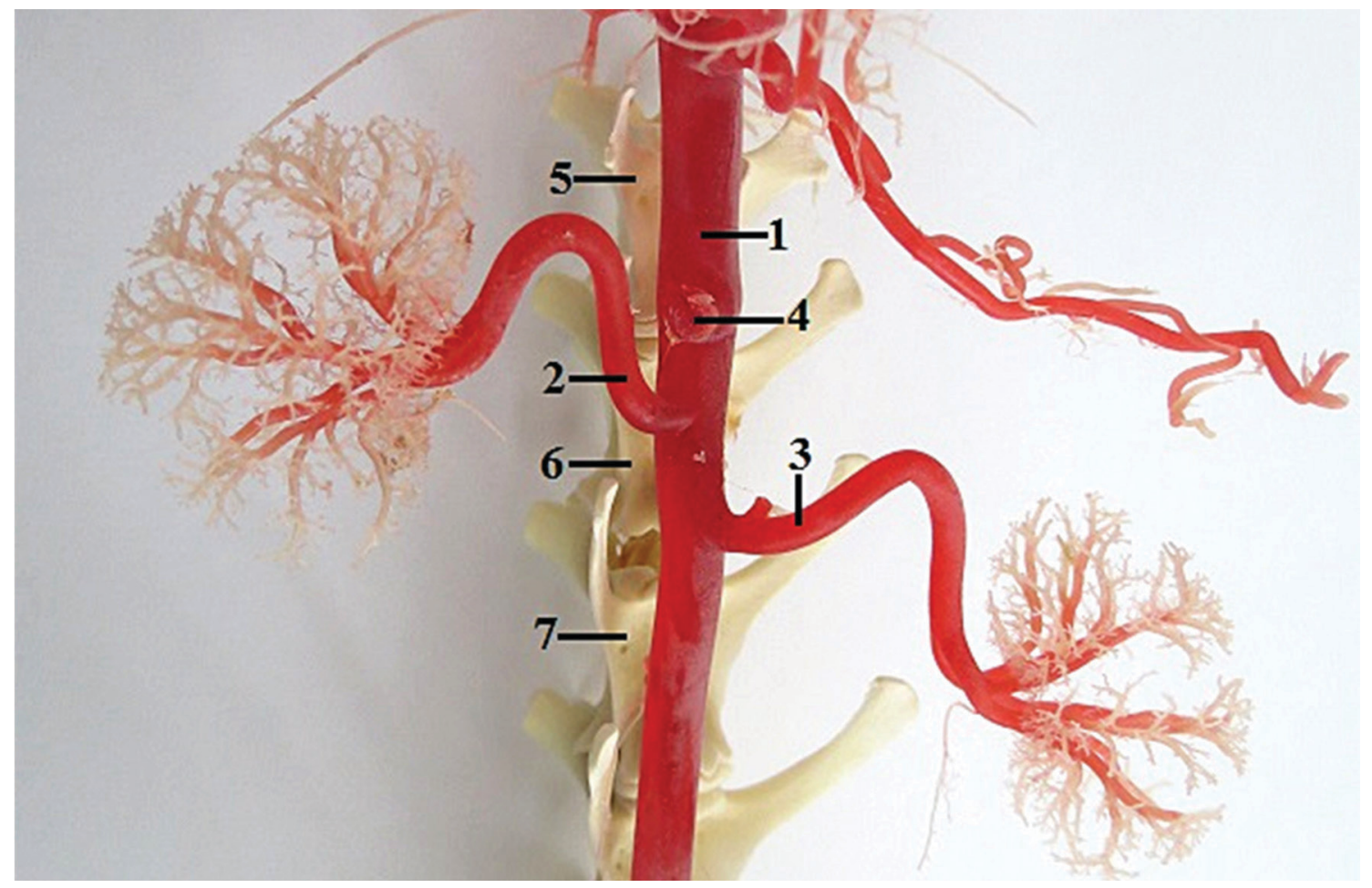

Fig. 1. Origin of the right and left renal arteries at the level of the second lumbar vertebra in the rabbit 1 - abdominal aorta; 2 - ight renal artery; 3 - left renal artery; 4 - cranial mesenteric artery; 5 - first lumbar vertebra; 6 - second lumbar vertebra; 7 - third lumbar vertebra. Macroscopic image, ventral view 


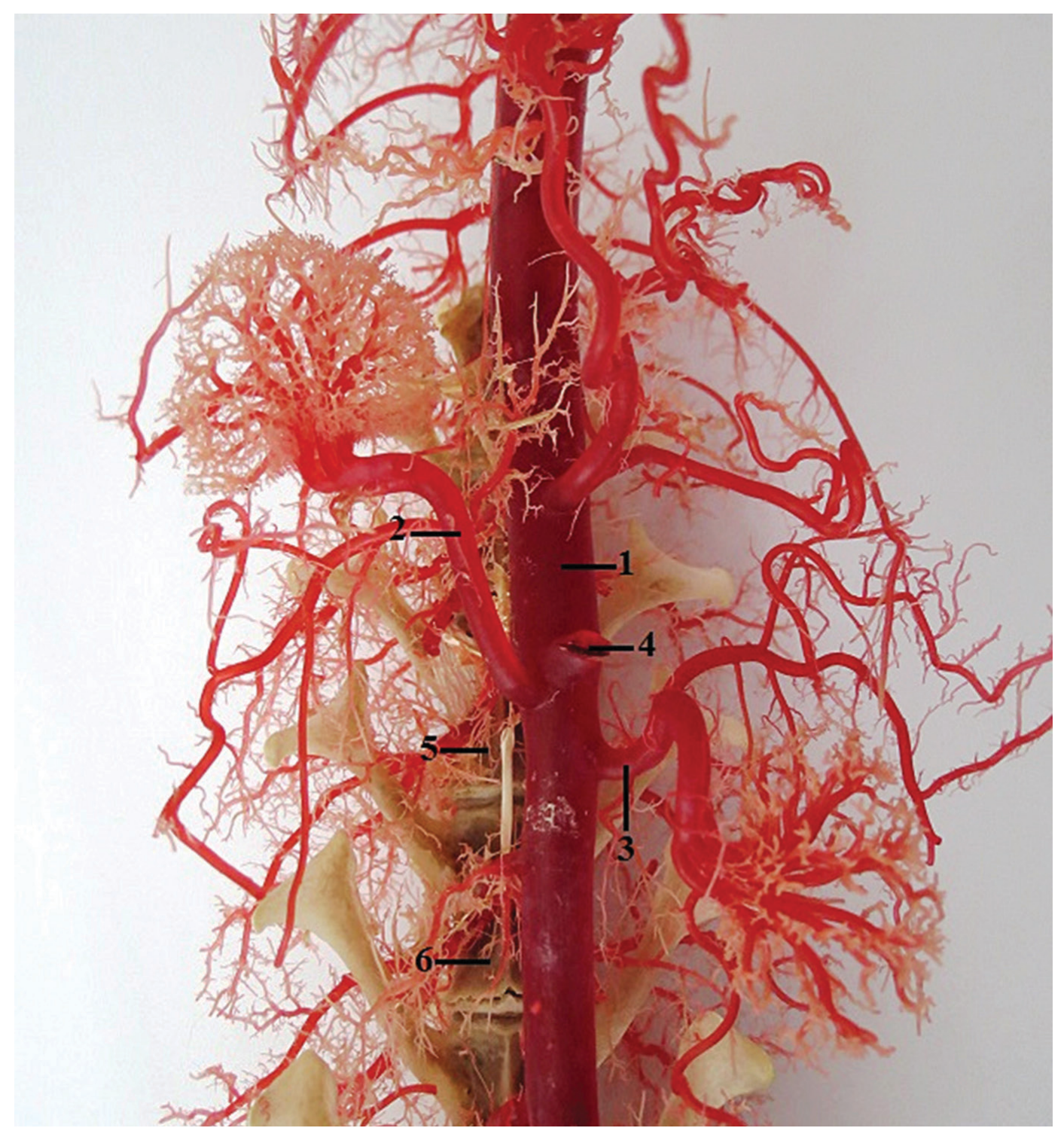

Fig. 2. Origin of the right and left renal arteries at the level of the second lumbar vertebra in the hare 1 - abdominal aorta; 2 - right renal artery; 3 - left renal artery;

4-cranial mesenteric artery; 5 - first lumbar vertebra; 6 - second lumbar vertebra. Macroscopic image, ventral view

divided into two or more branches, which gave rise to the interlobar arteries, which penetrated into the kidney.

In domesticated rabbit, the right renal artery originated at the level of the second lumbar vertebra in $70 \%$ of the cases: in $40 \%$ of the cases at the middle of the vertebral body (Fig. 1), in $20 \%$ of the cases at its cranial end and in $10 \%$ of the cases at its caudal end. In the remaining $30 \%$ of the cases, the origin was located at the level of the caudal end of the second lumbar vertebra. The origin of the left renal artery was located at the level of the second lumbar vertebra in $60 \%$ of the cases as follows: at the caudal end of the vertebral body in $40 \%$ of the cases (Fig. 1 ), at the cranial end in $10 \%$ of the cases and at the middle of the vertebral body in $10 \%$ of the cases. In $40 \%$ of the cases, the left renal artery arose at the level of the cranial end of the third lumbar vertebra.

In the European hare, the right renal artery arose at the level of the second lumbar vertebra: in $60 \%$ of the cases at the middle of the vertebral body (Fig. 2), in $30 \%$ of the cases at its cranial end and in $10 \%$ of the cases at the caudal end of the vertebral body. The left renal artery originated also at the level of the second lumbar vertebra. The origin was located at the level of the caudal end of the vertebral body in $70 \%$ of the cases (Fig. 2), in the middle of the vertebral body in $20 \%$ of the cases and at the cranial end in $10 \%$ of the cases. 


\section{DISCUSSION}

The general scheme of the arterial arrangement of rabbit kidneys is one independent single renal artery for each kidney. The renal arteries originate from the lateral surfaces of the abdominal aorta. The origin of the right renal artery is located cranially to the origin of the left renal artery [8, $10,15]$. We found the same arrangement on the corrosion casts in the rabbit and hare. Before entering the renal hilus, each of the renal arteries was divided into two or three interlobar arteries [9]. The cranial ureteral artery intended for the cranial part of the ureter was described as a branch arising from the corresponding renal artery [1]. The studies of aforementioned authors did not deal with the level of origin of the renal arteries from the abdominal aorta. The cranial ureteral artery was not present in our specimens.

Despite the frequent use of rabbits in experimental studies, the literature dealing with the vascular anatomy of the kidney in detail is still lacking. In the European rabbit, it has only been described as the presence of multiple renal arteries. The left renal artery has been described as double in $2.63 \%$ of the cases [11], while in our study we found a single left renal artery.

The anatomical variations of the renal arteries associated with several diseases are the purpose of different studies. The atypical anatomical arrangement of renal arteries is closely related to the obstructions of the urethra and blood pressure changes. The presence of variant renal arteries is of great significance in the renal vascular surgery. The presence of aberrant renal arteries limits the transplant [16].

\section{CONCLUSIONS}

The knowledge about the presence of anatomic variations of the renal arteries in experimental animals and related wild species is fundamental for academics-researchers and surgeons. This is the first work dealing with the description of renal arteries origin in the European hare of which we are aware.

\section{REFERENCES}

1. Douglas, C. G., Hossler, F.E., 1995: Vascular anatomy of the rabbit ureter. Anat. Rec., 242, 47-56.
2. Evan, A. P., Connors, B. A., Lingeman, J.E., Blomgren, P., Willis, L.R., 1996: Branching patterns of the renal artery of the pig. Anat. Rec., 246, 217-223.

3. Fernandez, F., Fernandez, G., Loske, A.M., 2009: Treatment time reduction using tandem shockwaves for lithotripsy: an in vivo study. J. Endourol., 23, 1247-1253.

4. Fuller, P.M., Huelke, D.F., 1973: Kidney vascular supply in rat, cat and dog. Acta Anat., 84, 516-522.

5. Groman, R. P., Bah, A., Berridge, B. R., Lees, G. E., 2004: Effects of serial ultrasound-guided renal biopsies on kidneys of healthy adolescent dogs. Vet. Radiol. Ultrasound., 45, 62-69.

6. Jain, R. K., Dhingra, L. D., Kumar, S., Sharma, D. N., 1985: Vascularization of kidneys in dogs (Canis familiaris). Indian J. Anim. Sci., 55, 406-409.

7. Kaouk, J.H., Gill, I.S., Desai, M.M., Banks, K.L., Raja, S.S., Skacel, M. et al., 2003: Laparoscopic anatrophic nephrolithotomy: feasibility study in a chronic porcine model. $J$. Urol., 169, 691.

8. Krause, W., 1884: The Anatomy of Rabbits. Processed in Topographical and Surgical Consideration (In German). Verlag von Wilhelm Engelmann, Leipzig, 258-263.

9. Mierzwa, J., 1975: The arterial system of kidneys in the rabbit. Folia Morphol., 34, 407-417.

10. Nejedlý, K., 1965: Biology and Systematic anatomy of Laboratory Animals (In Czech). SPN, Prague, 460-474.

11. Nowicki, W., Brudnicki, W., Iwanczyk, M., Jablonski, R., Skoczylas, B., 2010: Variation in Branches of the Abdominal Aorta in European Rabbit. EJPAU, 13, 10. http://www.ejpau. media.pl/volume13/issue4/art-10.html Accessed September 28, 2010.

12. Pereira-Sampaio, M. A., Favorito, L. A., Sampaio, F. J., 2004: Pig kidney: anatomical relationships between the intrarenal arteries and the kidney collecting system. Applied study for urological research and surgical training. J. Urol., 172, 20772081.

13. Pereira-Sampaio, M. A., Favorito, L. A., Henry, R. W., Sampaio, F. J., 2007: Proportional analysis of the pig kidney arterial segments. J. Endourol., 21, 784-788.

14. Pereira-Sampaio, M.A., Marques-Sampaio, B.P.S., Henry, R. W., Favorito, L. A., Sampaio, F. J. B., 2009: The dog kidney as experimental model in endourology: anatomic contribution. J. Endourol., 23, 989-993.

15. Popesko, P., Rajtova, V., Horak, J., 1990: Anatomic atlas of small laboratory animals I., 1st edn., Priroda, Bratislava, 255 pp.

16. Ramesh Rao, T., 2011: Aberrant renal arteries and its clinical significance: a case report. Int. J. Anat. Var., 4, 37-39. 
17. Shalgum, A., Marques-Sampaio, B. P. S., Dafalla, A., PereiraSampaio, M.A., 2011: Anatomical relationship between the collecting system and the intrarenal arteries in the rabbit: contribution as an experimental model. Anat. Histol. Embryol., 41, $130-138$

18. Styn, N. R., Wheat, J.C., Hall, T.L., Roberts, W. W., 2010: Histotripsy of VX- 2 tumor implanted in a renal rabbit model. J. Endourol., 24, 1145-1150.
19. Šulla, I. J., Lukáč, I., Šulla, I., Maršala, M., 2013: Experimental model of spinal cord compression injury in minipigs: A behavioral and MRI study. In Proceedings of the Annual Meeting: Evolution of Neurosurgery, San Francisco, USA, October $19-23,80$.

Received July 7, 2017

Accepted August 25, 2017 